\title{
Transfer of heat and hall current effects on peristaltic flow of a bingham fluid in a channel by Adomian decomposition method
}

\author{
Laxmi Devindrappa ${ }^{1}$, N. B. Naduvinamani ${ }^{2}$ \\ ${ }^{1}$ Gulbarga University, Kalaburagi-585106, India , ldb.biradar@gmail.com \\ ${ }^{2}$ Gulbarga University, Kalaburagi-585106, India, naduvinamaninb@yahoo.co.in
}

\begin{abstract}
This paper concerns with the transfer of heat and hall current effects on peristaltic flow of a Bingham fluid in a channel is analysis. Resulting differential equations after implementation of small Reynolds number and long wavelength considerations are numerically solved. The expressions for different parameters like pressure rise, flow rate and frictional force are determined. The effect of magnetic field, Darcy number, yield stress, amplitude ratio, Hall parameter and the temperature on pressure gradient, pumping characteristics and frictional force are discussed through nature of graphs.
\end{abstract}

Key words : Bingham fluid, peristaltic transport, hall Current, heat transfer and Adomian decomposition method.

\section{INTRODUCTION}

Peristaltic pumping has been the object of scientific and engineering researches during the recent past few decades. Latham [1] was probably the first to investigate the mechanism of peristalsis.

Peristaltic flow of MHD Jeffrey fluid in a vertical channel through Porous medium with Heat and Mass transfer with radiation analyzed by Eldabe et al. [2]. Nonlinear radiative peristaltic flow of hydromagnetic fluid with porous medium investigated by Hussain et al. [3]. Electro-Magnetohydrodynamic oscillatory flow of a dielectric fluid with heat transfer and porous medium was examined by Abo-elkhair [4].

The peristaltic transport of MHD Newtonian fluid with variable viscosity and heat transfer investigated by Nadeem and Akbar [5]. Heat transfer on the peristaltic transport of food bolus through oesophagus and wall properties obtained by Sreenadh et al. [6]. Effects of partial slip and heat transfer on peristaltic flow of a Williamson fluid in an inclined asymmetric channel studied by Akbar et al. [7]. Bhatti et al. [8] discussed the mathematical modeling of MHD peristaltic propulsion of two-phase flow through a Darcy-Brinkman Forchheimer porous medium with heat and mass transfer. Mekheimer et al. [9] studied the heat transfer on analytical investigation of peristaltic nanofluid flow in an asymmetric wavy wall channel. Peristaltic flow in a vertical asymmetric channel with effects of heat transfer and space porosity analysed by Mekheimer et al. [10]. Nadeem et al. [11] studied peristalsis with variable viscosity and influence of heat transfer.

If a particle is at rest in presence of magnetic field, the field has no effect. Similarly, if the particle path is in the same direction as that of the magnetic field, there is no effect i.e. the particle motion will be undeflected. But if the particle path has a component normal to the direction of magnetic field, the particle will be deflected. In presence of magnetic field of large extent at right angle to the direction of motion of a charged particle, the particle is deflected in a circular path. In addition to this deflection the particle experiences the electric field. The combined force acting on the particle is said to be Lorentz force. Abbasi et.al [12] have investigated the peristalsis of boron nitride-ethylene glycol nanofluid with temperature dependent thermal conductivity and hall effects . Interaction of pulsatile and peristaltic transport induced flows of a particle fluid suspension with Hall currents that had been examined by Gad [13]. Hussain et al. [14] studied the Heat and mass transfer effect in variable viscosity on peristaltic flow with Hall current and ion slip. Ellahi and Bhatti [15] analyzed peristaltic flow of Jeffrey fluid in a non-uniform rectangular duct under the effects of Hall and ion slip. Information on the topic is quite reasonable and researchers mention a few recent representative attempts and several useful references in their investigations[16-21].

The present study is transfer of heat and hall current effects on peristaltic flow of a bingham fluid in a channel under the supposition of long wavelength and low Reynolds number. The effects of various parameters are discussed through graphs in detail. 
Laxmi Devindrappa et al., International Journal of Emerging Trends in Engineering Research, 9(3), March 2021, 250 - 259

\section{MATHEMATICAL FORMULATION}

The wall deformation is given by

$H(X, t)=a+b \sin \frac{2 \pi}{\lambda}(x-c t)$

where $a$ half-width in a channel, $b$ is the amplitude, $\lambda$ the wavelength and $c$ is the wave speed. we cosider half-width of the channel as shown in the figure. Under the supposition that the channel length is an integral multiple of $\lambda$ and the pressure difference across the ends of the channel is a constant, the flow becomes steady in the wave frame $(x, y)$ moving with velocity $c$ away from the fixed frame $(X, Y)$ .The transformation between these two frames is given by $x=X-c t, y=Y, u(x, y)=U(X-c t, Y)$

and $w(x, y)=W(X-c t, Y)$

Where $U$ and $W$ are velocity in the laboratory frame and $u$ and $w$ are velocity in the wave frame.

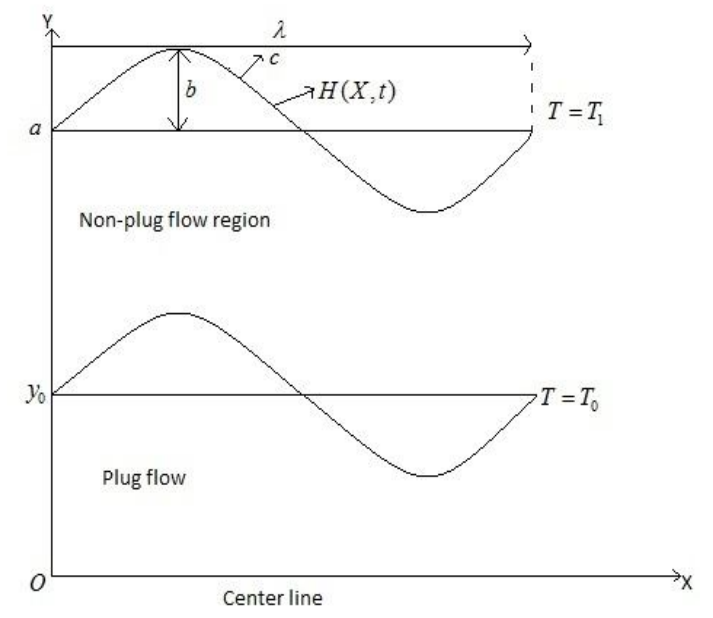

Figure 1: Physical model

The equations governing the flow are given by

$$
\begin{aligned}
& \frac{\partial u}{\partial x}+\frac{\partial w}{\partial y}=0 . \\
& \rho\left(u \frac{\partial u}{\partial x}+w \frac{\partial u}{\partial y}\right)=-\frac{\partial p}{\partial x}+\frac{\partial \tau_{x x}}{\partial x}+\frac{\partial \tau_{y x}}{\partial x} \\
& +\frac{\sigma B_{0}{ }^{2}}{1+m^{2}}(-u+m w)-\frac{\mu}{k}(u+c)+\rho g \alpha\left(F-F_{0}\right) .
\end{aligned}
$$

Where $m=\frac{\sigma B_{0}}{e n}$ is the hall term, e-Electronic charge, n-number density of electrons and $\tau_{i j}$ denote the stresses.

$$
\begin{gathered}
\tau_{i j}=\left(\mu+\frac{\tau_{y}}{\dot{\gamma}}\right) \dot{\gamma}_{i j} \text { for } \tau \geq \tau_{y} \\
\tau_{i j}=\dot{\gamma}_{i j}=0 \quad \text { for } \quad \tau<\tau_{y}
\end{gathered}
$$

Where $\gamma_{i j}$ is the rate of strain tensor,

$$
\begin{aligned}
& \dot{\gamma}_{i j}=\frac{\partial u_{i}}{\partial x_{j}}+\frac{\partial u_{j}}{\partial x_{i}}, \\
& \tau=\sqrt{\frac{1}{2} \tau_{i j} \tau_{i j}} \\
& \dot{\gamma}=\sqrt{\frac{1}{2} \dot{\gamma}_{i j} \dot{\gamma}_{i j}}=\sqrt{\left(\frac{\partial u}{\partial x}\right)^{2}+\left(\frac{\partial w}{\partial y}\right)^{2}+\left(\frac{\partial u}{\partial y}+\frac{\partial w}{\partial x}\right)^{2}}
\end{aligned}
$$

Introducing the non-dimensional quantities

$$
\begin{aligned}
& x=\frac{x}{\lambda}, y=\frac{y}{a}, u=\frac{u}{c}, w=\frac{w}{c \delta}, \delta=\frac{a}{\lambda}, p=\frac{p a^{2}}{\mu c \lambda}, \\
& t=\frac{c t}{\lambda}, h=\frac{H}{a}, \phi=\frac{b}{a}, \tau_{y}=\frac{a \tau_{y}}{\lambda}, y_{0}=\frac{y_{0}}{a}, \\
& \Theta=\frac{F-F_{0}}{F_{1}-F_{0}}, \alpha=\frac{Q_{0} a^{2}}{k\left(F_{1}-F_{0}\right)}, G_{r}=\frac{\rho \alpha g a^{2}\left(F_{1}-F_{0}\right)}{\mu c}, \\
& p_{r}=\frac{\mu c_{p}}{k}, M=\sqrt{\frac{\rho}{\mu}} a B_{0}, \operatorname{Re}=\frac{\rho c a}{\mu}, D a=\frac{k}{a^{2}} .
\end{aligned}
$$

Into equations, we get

$$
\frac{\partial u}{\partial x}+\frac{\partial w}{\partial y}=0
$$


$\operatorname{Re} \delta\left(u \frac{\partial u}{\partial x}+w \frac{\partial u}{\partial y}\right)=-\frac{\partial p}{\partial x}+\delta^{2} \frac{\partial \tau_{x x}}{\partial x}+$

$\frac{\partial \tau_{y x}}{\partial y}-\left(\frac{M^{2}}{1+m^{2}}+\frac{1}{D a}\right)(u+1)+G_{r} \Theta$.

$\operatorname{Re} \delta^{3}\left(u \frac{\partial v}{\partial x}+w \frac{\partial v}{\partial y}\right)=-\frac{\partial p}{\partial y}+$

$\delta^{2} \frac{\partial \tau_{x y}}{\partial x}+\delta \frac{\partial \tau_{y y}}{\partial y}-\frac{\delta^{2}}{D a} w$.

$\operatorname{Re} P_{r} \delta\left(u \frac{\partial}{\partial x}+w \frac{\partial}{\partial y}\right) \Theta=\delta^{2} \frac{\partial^{2} \Theta}{\partial x^{2}}+\frac{\partial^{2} \Theta}{\partial y^{2}}+\alpha$.

(14)

where

$$
\begin{aligned}
& \tau_{i j}=\left(\mu+\frac{\tau_{y}}{\dot{\gamma}}\right) \dot{\gamma}_{i j} \text { for } \tau \geq \tau_{y} \\
& \tau_{i j}=\dot{\gamma}_{i j}=0 \text { for } \tau<\tau_{y} . \\
& \dot{\gamma}_{x y}=\dot{\gamma}_{y x}=\frac{\partial u}{\partial y}+\delta^{2} \frac{\partial u}{\partial x} . \\
& \dot{\gamma}_{x x}=2 \delta \frac{\partial u}{\partial x} \dot{\gamma}_{y y}=2 \delta \frac{\partial w}{\partial y} . \\
& \dot{\gamma}=\sqrt{2 \delta^{2}\left(\left(\frac{\partial u}{\partial x}\right)^{2}+\left(\frac{\partial w}{\partial y}\right)^{2}\right)+\left(\frac{\partial u}{\partial y}+\delta^{2} \frac{\partial w}{\partial x}\right)^{2}} \\
& \tau=\sqrt{\tau_{x y}^{2}+\delta^{2} \tau^{2}}
\end{aligned}
$$

Under the assumptions of long wavelength and low Reynolds number, the equations (12) to (14) reduce to

$0=-\frac{\partial p}{\partial x}+\frac{\partial \tau_{y x}}{\partial y}-N^{2}(u+1)+G_{r} \Theta$.

Where $N^{2}=\frac{M^{2}}{1+m^{2}}+\frac{1}{D a}$

$0=-\frac{\partial p}{\partial y}$.

$\frac{\partial^{2} \Theta}{\partial y^{2}}+\alpha=0$.

$\tau_{x y}=\frac{\partial u}{\partial y}-\tau_{y}$ for $\tau \geq \tau_{y}$.

$\tau_{x y}=0$ for $\tau<\tau_{y}$.

Here $\tau_{y}$ is the yield stress.
Here equation (22) indicates that $p$ is independent of $y$ and depends on $x$. Therefore, Eq. (21), can be rewritten as

$\frac{\partial^{2} u}{\partial y^{2}}-N^{2} u=\frac{d p}{d x}+N^{2}+G_{r} \Theta$.

The non-dimensional boundary conditions are

$\frac{\partial u}{\partial y}=\tau_{y}$ at $y=0$.

$u=-1$ at $y=h$.

$\frac{\partial \Theta}{\partial y}=0 \quad$ at $\quad y=0 \quad$ and $\Theta=1 \quad$ at $\quad y=h$ (29)

The volume flux $q$ through each cross section in the wave frame is given by

$q=\int_{0}^{y_{0}} u_{p} d y+\int_{y_{0}}^{h} u d y$

The volume flow rate $Q(X, t)$ in the laboratory frame between the centre line and the wall is

$Q(X, t)=\int_{0}^{H} U d y=\int_{0}^{h}(u+1) d y=q+h$

\section{SOLUTION OF THE PROBLEM}

The temperature Eq. (23) with the boundary conditions (29) gives

$\Theta=1+\frac{\alpha}{2}\left(h^{2}-y^{2}\right)$.

We use Adomian decomposition method, we write Eq. (26) in the form

$L_{y y} u-N^{2} u=\frac{d p}{d x}+N^{2}+G_{r} \Theta$.

Putt Eq. (32) in Eq. (33), we get

$L_{y y} u-N^{2} u=\frac{d p}{d x}+N^{2}+G_{r}\left(1+\frac{\alpha}{2}\left(h^{2}-y^{2}\right)\right)$

Where $L=\frac{\partial^{2}}{\partial y^{2}}$ Since $L$ is differential operator, $L^{-1}$ is integration operator defined by:

$$
L^{-1}(.)=\int_{0}^{y} \int_{0}^{y}(.) d y d y \text {. }
$$

Eq. (34) becomes

$u=c_{1}+c_{2} y+L^{-1}\left(\begin{array}{l}\frac{d p}{d x}+N^{2}+G_{r} \\ \left(1+\frac{\alpha}{2}\left(h^{2}-y^{2}\right)\right)\end{array}\right)$

$+L^{-1}\left(N^{2} u\right)$. 
Laxmi Devindrappa et al., International Journal of Emerging Trends in Engineering Research, 9(3), March 2021, 250 - 259

The function can be determined by using the boundary conditions (27-28). one can write:

$u=\sum_{n=0}^{\infty} u_{n}$.

From eq. (36)

$$
\begin{aligned}
& u_{0}=c_{1}+c_{2} y+\left(\frac{d p}{d x}+N^{2}+G_{r} \frac{\alpha h^{2}}{2}+G_{r}\right) \\
& \frac{y^{2}}{2 !}-G_{r} \alpha \frac{y^{4}}{4 !} . \\
& u_{n+1}=N^{2} L^{-1}\left(u_{n}\right), \quad n \geq 0 .
\end{aligned}
$$

Using conditions (27-28) and from (33) and (37), we write

$$
\begin{aligned}
& u_{1}=c_{1} \frac{(N y)^{2}}{2 !}+\frac{c_{2}}{N} \frac{(N y)^{3}}{3 !}+\left(\frac{d p}{d x}+N^{2}\right) \frac{1}{N^{2}} \frac{(N y)^{4}}{4 !} \\
& +G_{r}\left(1+\frac{\alpha h^{2}}{2}\right) \frac{1}{N^{2}} \frac{(N y)^{4}}{4 !}-\frac{G_{r} \alpha}{N^{4}} \frac{(N y)^{6}}{6 !} . \\
& u_{2}=c_{1} \frac{(N y)^{4}}{4 !}+\frac{c_{2}}{N} \frac{(N y)^{5}}{5 !}+\left(\frac{d p}{d x}+N^{2}\right) \frac{1}{N^{2}} \frac{(N y)^{6}}{6 !} \\
& +G_{r}\left(1+\frac{\alpha h^{2}}{2}\right) \frac{1}{N^{2}} \frac{(N y)^{6}}{6 !}-\frac{G_{r} \alpha}{N^{4}} \frac{(N y)^{8}}{8 !}
\end{aligned}
$$$$
u_{n}=c_{1} \frac{(N y)^{2 n-2}}{2 n !}+\frac{c_{2}}{N} \frac{(N y)^{2 n-1}}{(2 n-1) !}+\left(\frac{d p}{d x}+N^{2}\right) \frac{1}{N^{2}}
$$$$
\frac{(N y)^{2 n}}{(2 n) !}+G_{r}\left(1+\frac{\alpha h^{2}}{2}\right) \frac{1}{N^{2}} \frac{(N y)^{2 n}}{(2 n) !}-\frac{G_{r} \alpha}{N^{4}} \frac{(N y)^{2 n+2}}{(2 n+2) !} \text {. }
$$$$
u=c_{1} \cosh (N y)+\frac{c_{2}}{N} \sinh (N y)+
$$$$
\left(\left(\frac{d p}{d x}+N^{2}\right)\left(1+\alpha \frac{h^{2}}{2}\right) \frac{1}{N^{2}}\right)(\cosh (N y)-1)
$$$$
-\frac{G_{r} \alpha}{N^{4}}\left(\cosh (N y)-1-\frac{(N y)^{2}}{2 !}\right) \text {. }
$$

This may be simplified as:

$$
\begin{aligned}
& u=\frac{1}{N^{2}}\left(\frac{d p}{d x}+G_{r}\right)\left(\frac{\cosh (N y)}{\cosh (N h)}-1\right)- \\
& \frac{\tau_{y}}{N} \tanh (N h) \cosh (N y)+\frac{\tau_{y}}{N} \sinh (N y)-1- \\
& G_{r} \alpha\left(\frac{1}{N^{4}} \frac{\cosh (N y)}{\cosh (N h)}+\frac{h^{2}}{2 N^{2}}-\frac{1}{N^{2}}-\frac{y^{2}}{2}\right) .
\end{aligned}
$$

The upper limit of plug flow region using the boundary condition

$$
\frac{\partial u}{\partial y}=0 \quad \text { at } \quad y=y_{0} .
$$

We have

$$
\begin{aligned}
& \tau_{y}=-\frac{1}{N}\left(G_{r}+\frac{d p}{d x}\right)\left(\frac{\sinh \left(N y_{0}\right)}{\cosh N\left(h-y_{0}\right)}\right) \\
& +\frac{G_{r} \alpha}{N^{3}}\left(\frac{-N^{3} y_{0}+\sinh \left(N y_{0}\right)}{\cosh N\left(h-y_{0}\right)}\right) .
\end{aligned}
$$

Taking $y=y_{0}$ in Eq. (38), the velocity in plug flow region as

$$
\begin{aligned}
& u_{p}=\frac{1}{N^{2}}\left(\frac{d p}{d x}+G_{r}\right)\left(\frac{1-\cosh \left(N\left(h-y_{0}\right)\right)}{\cosh \left(N\left(h-y_{0}\right)\right)}\right) \\
& -1-\frac{G_{r} \alpha}{N^{4}}\left(\frac{1-N^{3} y_{0} \tanh \left(N\left(h-y_{0}\right)\right)}{\cosh \left(N\left(h-y_{0}\right)\right)}\right) \\
& -G_{r} \alpha\left(\frac{h^{2}}{2 N^{2}}-\frac{1}{N^{2}}-\frac{y_{0}^{2}}{2}\right) .
\end{aligned}
$$

The volume flux $q$ through each cross section is given by

$$
\begin{aligned}
& q=\int_{0}^{y_{0}} u_{p} d y+\int_{y_{0}}^{h} u d y=-h+y_{0}+y_{0}\left(A_{1}-A_{2}-A_{3}\right) \\
& +\frac{1}{6 N^{5}}\left(G_{r}\left(A_{4} y_{0}-A_{5}\right)\right)+\frac{d p}{d x}\left(-\frac{h}{N^{2}}+\frac{y_{0}}{N^{2}}+A_{6}\right) .
\end{aligned}
$$

Where

$$
\begin{aligned}
& A_{1}=-1+\frac{\left(1-\cosh \left(N\left(h-y_{0}\right)\right)\right) \operatorname{sech}\left(N\left(h-y_{0}\right)\right)}{N^{2}} . \\
& A_{2}=\frac{\alpha \operatorname{sech}\left(N\left(h-y_{0}\right)\right) G_{r}\left(1-N^{3} \sinh \left(N\left(h-y_{0}\right)\right) y_{0}\right)}{N^{4}} . \\
& A_{3}=\alpha G_{r}\left(-\frac{1}{N^{2}}+\frac{h^{2}}{2 N^{2}}-\frac{y_{0}^{2}}{2}\right) . \\
& A_{4}=h N^{3}\left(-6+\left(6+h^{2}\left(-3+N^{2}\right)\right) \alpha\right)+ \\
& 3 N^{3}\left(\begin{array}{l}
\left.2+\left(-2+h^{2}\right) \alpha-2 \alpha \sec h(h N)\right) \\
\left(-1+\sec h\left(N\left(h-y_{0}\right)\right)\right)
\end{array}\right) .
\end{aligned}
$$


$A_{5}=N^{5} \alpha y_{0}{ }^{3}-6\left(N^{2}-\alpha\right)\left(\begin{array}{l}\sec h(h N) \sec h\left(N\left(h-y_{0}\right)\right) \\ \sin h\left(N y_{0}\right)-\tanh (h N)\end{array}\right)$.

$A_{6}=\frac{\left\{\begin{array}{l}-\sec h(h N) \sec h\left(N\left(h-y_{0}\right)\right) \\ \sinh \left(N y_{0}\right)+\tanh (h N)\end{array}\right\}}{N^{3}}$.

From Eq. (41) we have

$\frac{d p}{d x}=\frac{q-\left(\begin{array}{l}-h+y_{0}+y_{0}\left(A_{1}-A_{2}-A_{3}\right)+ \\ \frac{1}{6 N^{5}}\left(G_{r}\left(A_{4} y_{0}-A_{5}\right)\right)\end{array}\right)}{\left(-\frac{h}{N^{2}}+\frac{y_{0}}{N^{2}}+A_{6}\right)}$

The pressure rise and frictional force are given by

$$
\begin{aligned}
& \Delta p=\int_{0}^{1} \frac{d p}{d x} d x \\
& F=\int_{0}^{1} h\left(-\frac{d p}{d x}\right) d x
\end{aligned}
$$

\section{RESULTS AND DISCUSSION}

(Figures. 2-7) show the axial pressure gradient $\frac{d p}{d x}$ with respect to the axial $x$ for different values of $\phi, D_{a}, M$, $y_{0}$, Grashof number $G_{r}$, Hall parameter $m$ and heat parameter $\alpha$ in channel. From Figure. 2 it is analyzed that, the pressure gradient escalates with increase in amplitude ratio $\phi$. From Figure. 3 it is seen that, the pressure gradient increases with increase in Hartmann number. From Figure. 4 it is noticed that, the pressure gradient diminishes with increase in Hall parameter. From Figure. 5 it is observed that, the pressure gradient diminishes with increase in $D_{a}$. Further from Figure. 6 the pressure gradient is elevated with the increasing heat parameter $\alpha$. From Figure. 7 it is seen that, the pressure gradient escalates with increase $G_{r}$. (Figures. 8-13) shows the volumetric flow rate, it is observed that pumping is low when compared to Newtonian fluid. From Figure. 8 revealed the with an escalates in $y_{0}$ there is diminishes in the region $\Delta p>0$, no changes in region $\Delta p=0$ and increases in region $\Delta p<0$. From Figure. 9 it is revealed that with an increase in $M$ there is increase in the region $\Delta p>0$, the free pumping region $\Delta p=0$ and diminishes in the augmented pumping region $\Delta p<0$. It can be noticed from Figure. 10 increase in Hall parameter $m$ causes the decreases in the pumping region $\Delta p>0$, no changes in the free pumping region $\Delta p=0$ and increases in the augmented pumping region $\Delta p<0$. From Figure. 11 revealed thet with an increase in $D a$ there is decreases in the pumping region $\Delta p>0$, no changes in the free pumping region $\Delta p=0$ and increases in the augmented pumping region $\Delta p<0$. The effect of heat parameter $\alpha$ on pumping is depicted in Figure. 12 it is noted that $\alpha$ elevates pressure differences with increasing averaged volumetric flow rate in the pumping region $\Delta p>0$, the free pumping region $\Delta p=0$ and the augmented pumping region $\Delta p<0$. Figure. 13 it is noted that $G_{r}$ parameter significantly decreases pressure differences with increasing averaged volumetric flow rate in the pumping region $\Delta p>0$, the free pumping region $\Delta p=0$ and the augmented pumping region $\Delta p<0$. (Figures. 14-19) frictional forces have opposite behavior to the pressure rise. The effects of temperature field for values of $m, D_{a}$ and $M$, we have plotted (Figure. 20-22) it is observed that with the increase of $m$ and $D_{a}$, the temperature field increases. Further, it is seen that temperature field diminishes with increase in $M$.

\section{CONCLUSION}

Transfer of heat and hall current effects on peristaltic flow of a bingham fluid in a channel is analysed. Graphs were plotted in order to analyze the effects of various physical parameters. Main observations of the analysis are below.

- Pressure gradient increases with the increase of $\phi$, $M$, Grashof number $G_{r}$, and heat parameter $\alpha$ in asymmetric channel. while it diminishes by increasing $m$ and $D a$.

- Pressure rise diminishes ...with the increase in Hall parameter $m$. However it increases with an increase in $M, \alpha$.

- With the increase of $m$ the temperature field increases. Further, it is seen that temperature field decreases with increase in $M$. 
Laxmi Devindrappa et al., International Journal of Emerging Trends in Engineering Research, 9(3), March 2021, 250 - 259

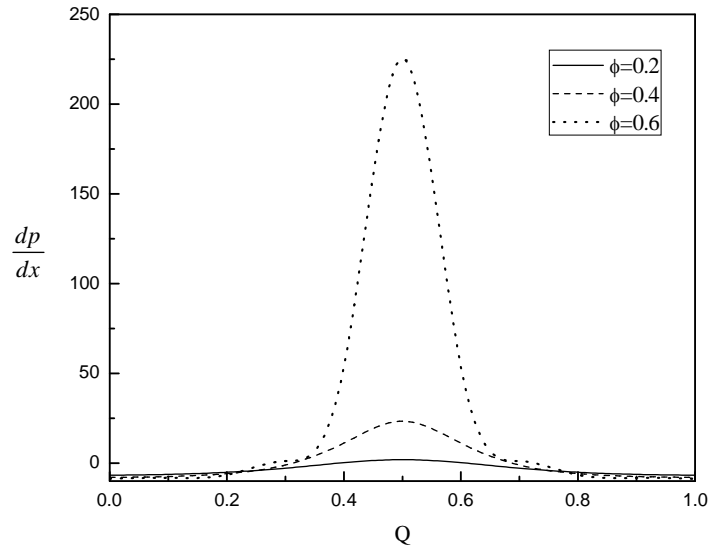

Figure 2: Pressure gradient with $\phi$ for

$G_{r}=3, \alpha=0.1, M=2, y_{0}=0.2, m=1$ and $D a=0.1$

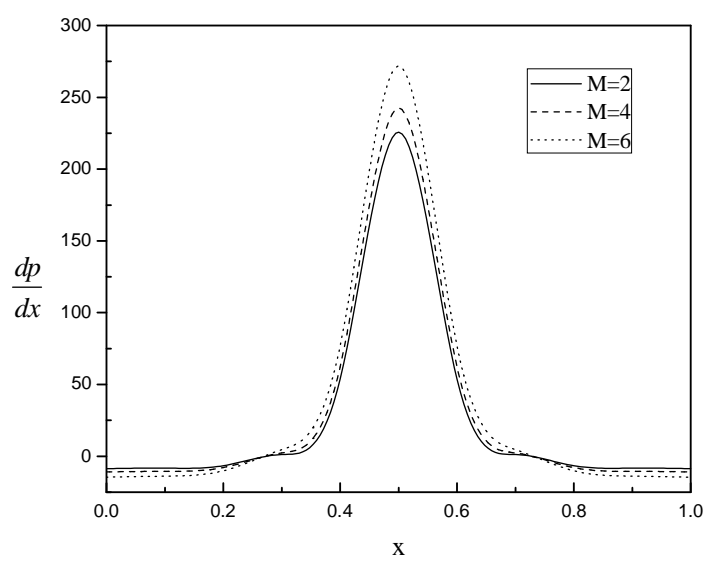

Figure 3: Pressure gradient with $M$ for $G_{r}=3, \alpha=0.1, \phi=0.6, y_{0}=0.2, m=1$ and

$$
D a=0.1
$$

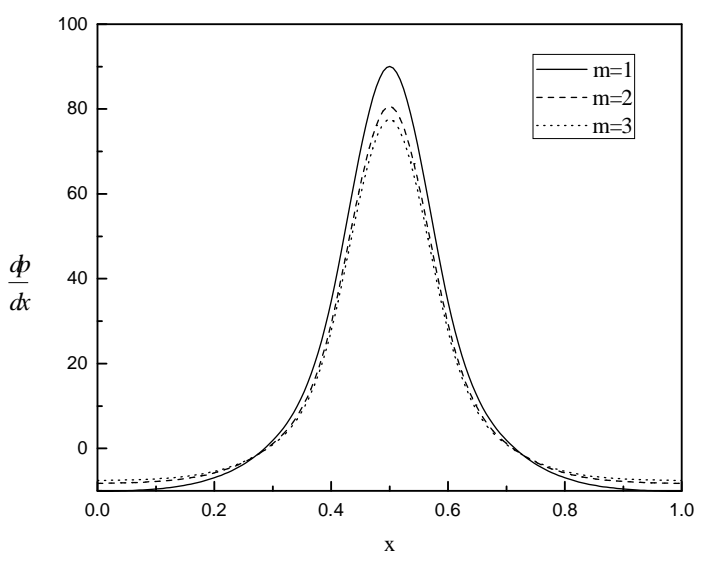

Figure 4: Pressure gradient with $m$ for

$$
\begin{gathered}
G_{r}=2, \alpha=0.1, \phi=0.6, y_{0}=0.2, M=4 \text { and } \\
D a=0.1
\end{gathered}
$$

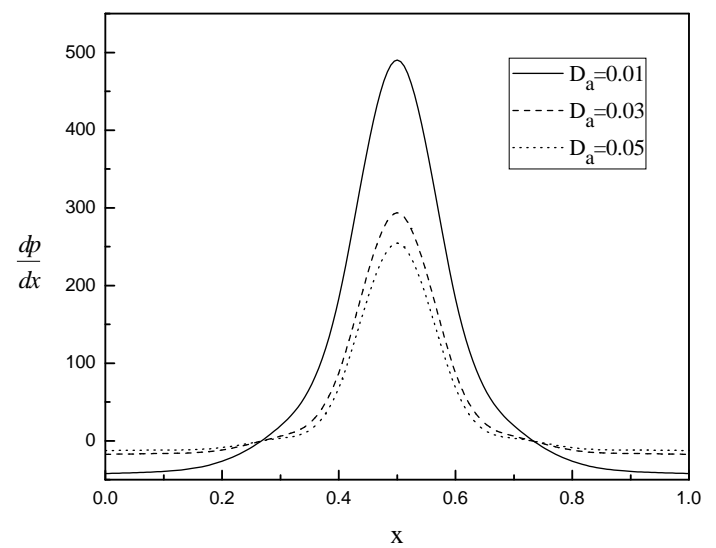

Figure 5: Pressure gradient with $D a$ for $G_{r}=3, \alpha=0.1, \phi=0.6, y_{0}=0.2, m=1$ and $M=2$

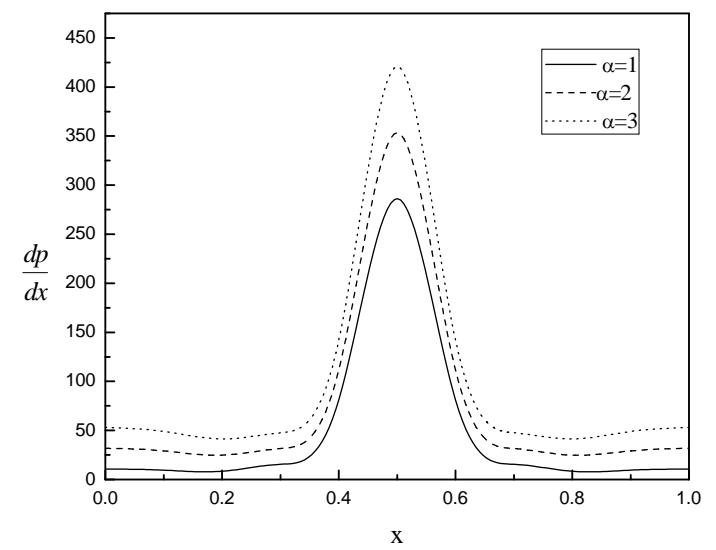

Figure 6: Pressure gradient with $\alpha$ for $G_{r}=3, M=2, \phi=0.6, y_{0}=0.2, m=1$ and $D a=0.1$ 


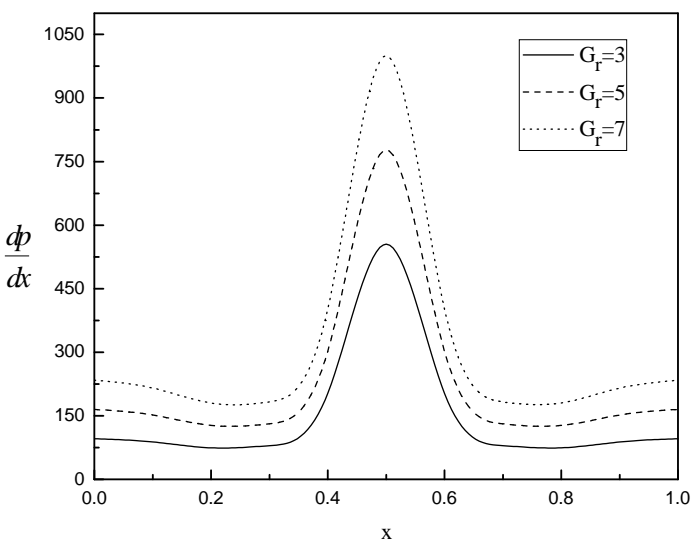

Figure 7: Pressure gradient with $G_{r}$ for $\alpha=5, M=2, \phi=0.6, y_{0}=0.2, m=1$ and $D a=0.1$

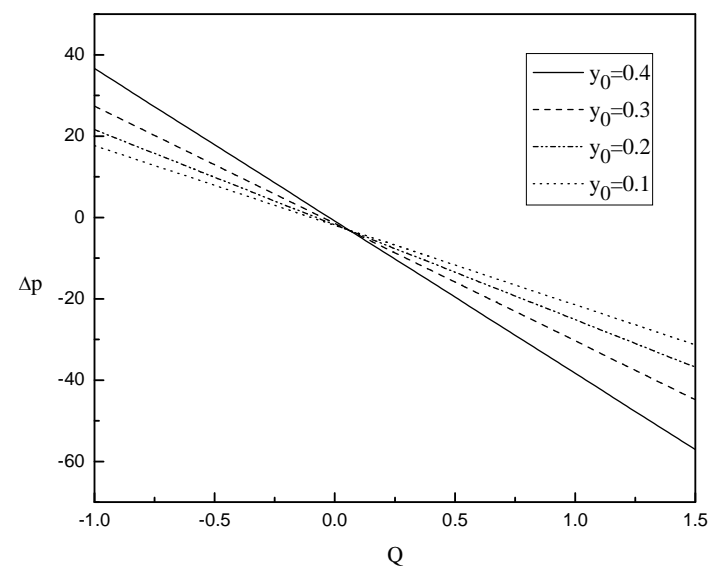

Figure 8: Pressure rise with time-averaged flux for different values of $y_{0}$ for $\alpha=0.1, M=2, \phi=0.6, G_{r}=3, m=1$ and

$$
D a=0.1
$$

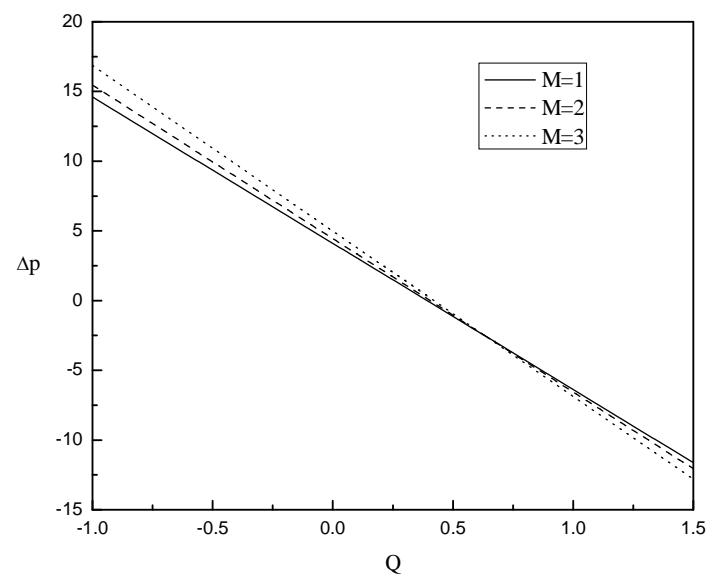

Figure 9: Pressure rise with time-averaged flux for different values of $M$ for $\alpha=1, y_{0}=0.2, \phi=0.6, G_{r}=2, m=2$ and $D a=0.1$

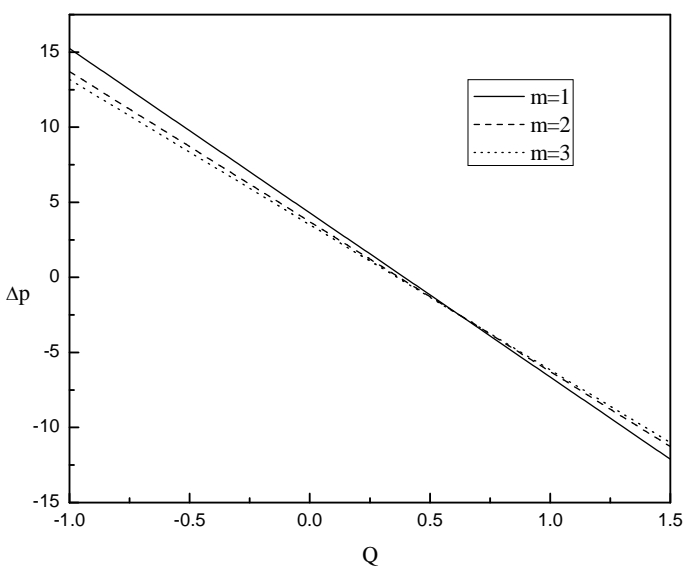

Figure 10: Pressure rise with time-averaged flux for different values of $m$ for $\alpha=1, y_{0}=0.1, \phi=0.6, G_{r}=2, M=2$ and $D a=0.1$

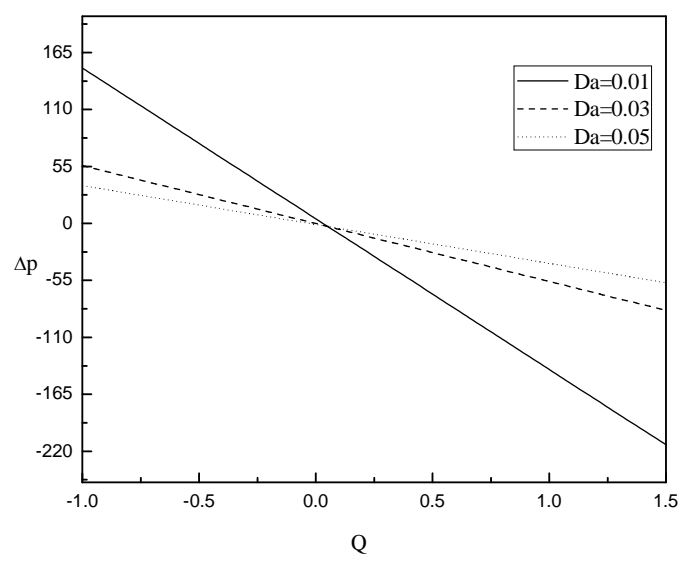

Figure 11: Pressure rise with time-averaged flux for different values of $D a$ for $\alpha=0.1, y_{0}=0.2, \phi=0.6, G_{r}=3, m=1$ and $M=2$ 


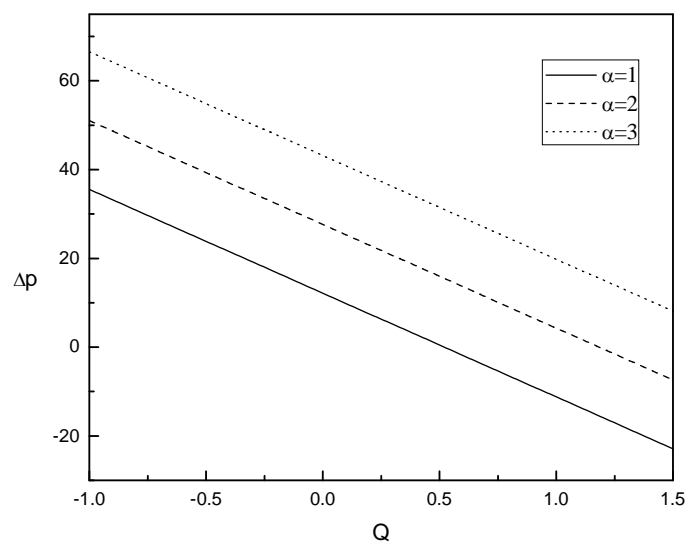

Figure 12: Pressure rise with time-averaged flux for different values of $\alpha$ for $M=2, y_{0}=0.2, \phi=0.6, G_{r}=3, m=1$ and $D a=0.1$

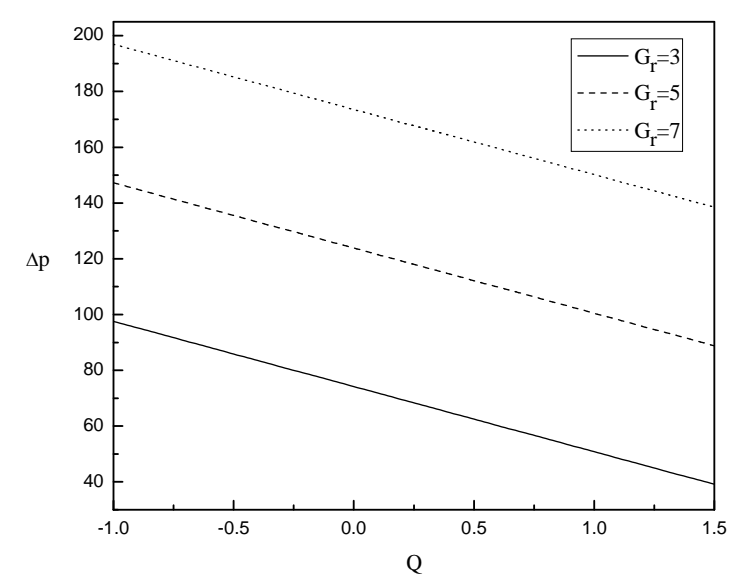

Figure 13: Pressure rise with time-averaged flux for different values of $G_{r}$ for $M=2, y_{0}=0.2, \phi=0.6, \alpha=5, m=1$ and $D a=0.1$

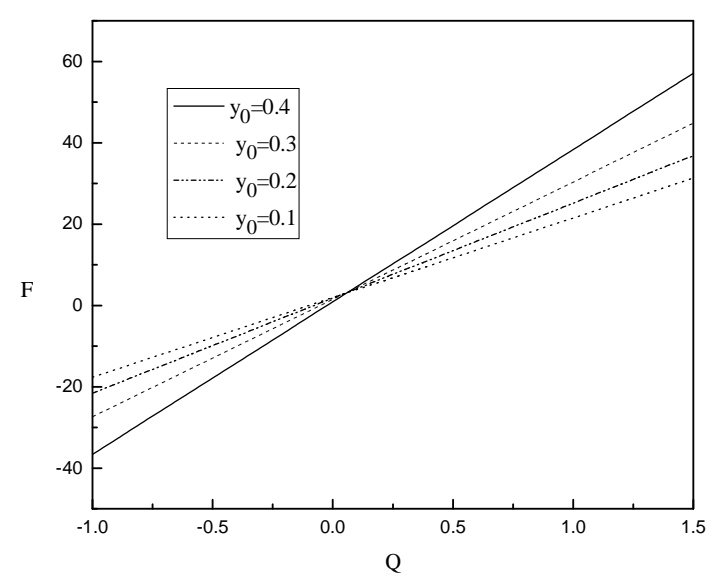

Figure 14: Frictional force with time-averaged flux for different values of $y_{0}$ for $M=2, G_{r}=3, \phi=0.6, \beta=0.1, m=1$ and $D a=0.1$

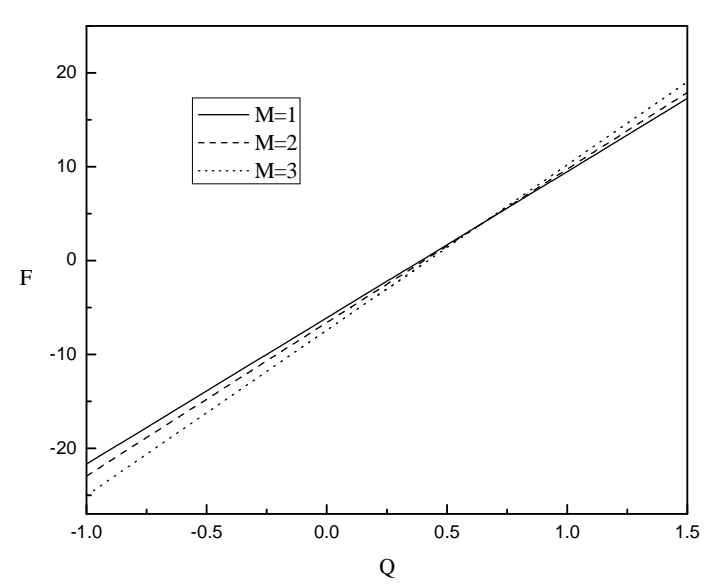

Figure 15. Frictional force with time-averaged flux for different values of $M$ for $\phi=0.6, G_{r}=2, y_{0}=0.2, \alpha=1, m=2$ and $D a=0.1$

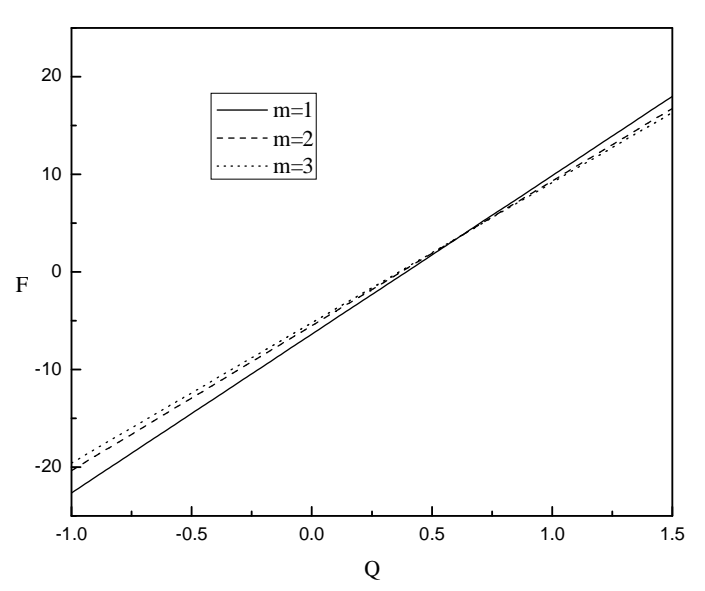

Figure 16: Frictional force with time-averaged flux for different values of $m$ for $\phi=0.6, G_{r}=2, y_{0}=0.1, \alpha=1, M=2$ and $D a=0.1$ 
Laxmi Devindrappa et al., International Journal of Emerging Trends in Engineering Research, 9(3), March 2021, 250 - 259

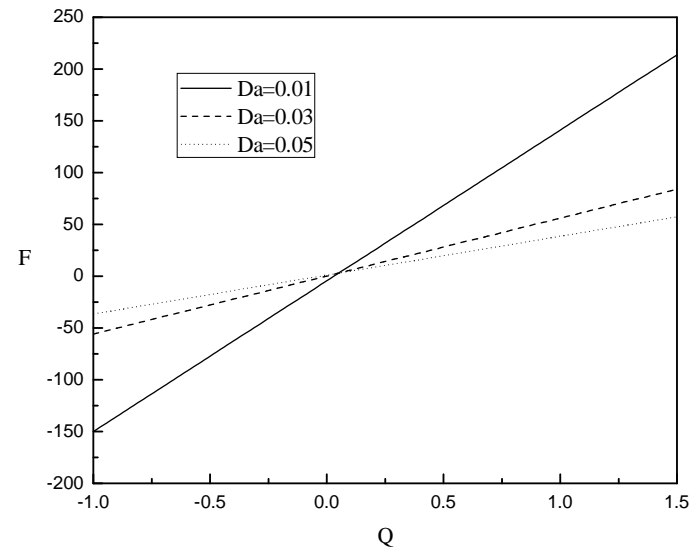

Figure 17: Frictional force with time-averaged flux for different values of $D a$ for $\phi=0.6, G_{r}=3, y_{0}=0.2, \alpha=0.1, m=1$ and $M=2$

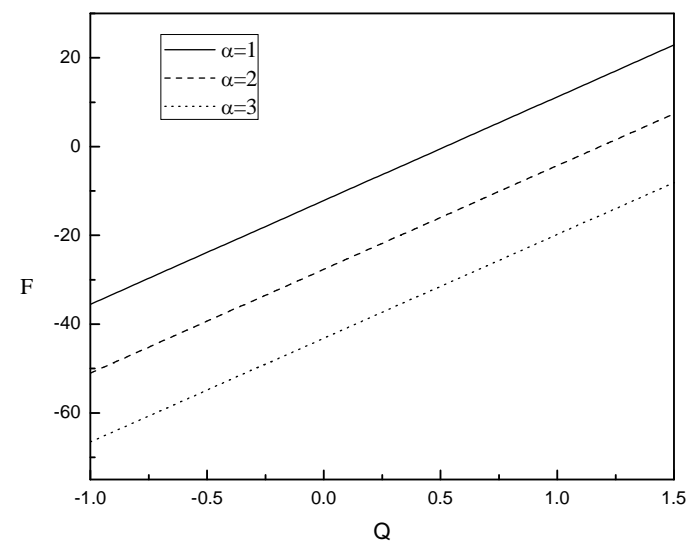

Figure 18: Frictional force with time-averaged flux for different values of $\alpha$ for $\phi=0.6, G_{r}=3, y_{0}=0.2, M=2, m=1$ and $D a=0.1$

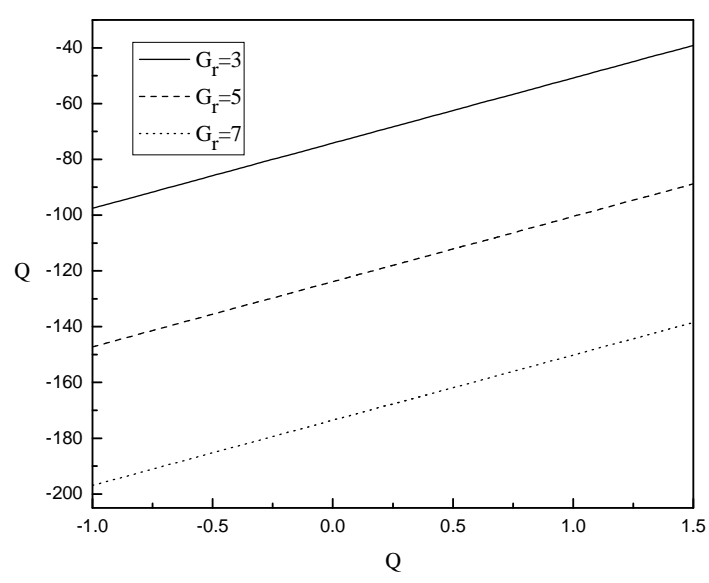

Fig.19. Frictional force with time-averaged flux for different values of $G_{r}$ for $\phi=0.6, \alpha=5, y_{0}=0.2, M=2, m=1$ and $D a=0.1$

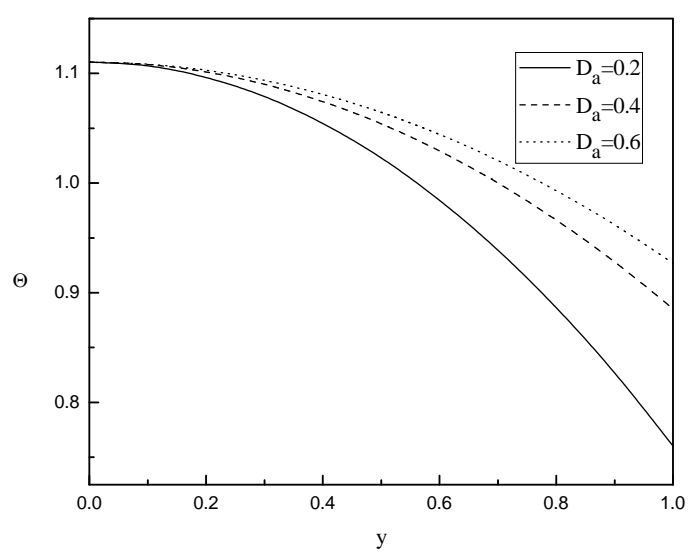

Figure 20: Temperature field for different values of $D a$, the other parameters are

$\phi=0.6, G_{r}=3, y_{0}=0.2, M=2, m=1$ and $\alpha=0.1$ 


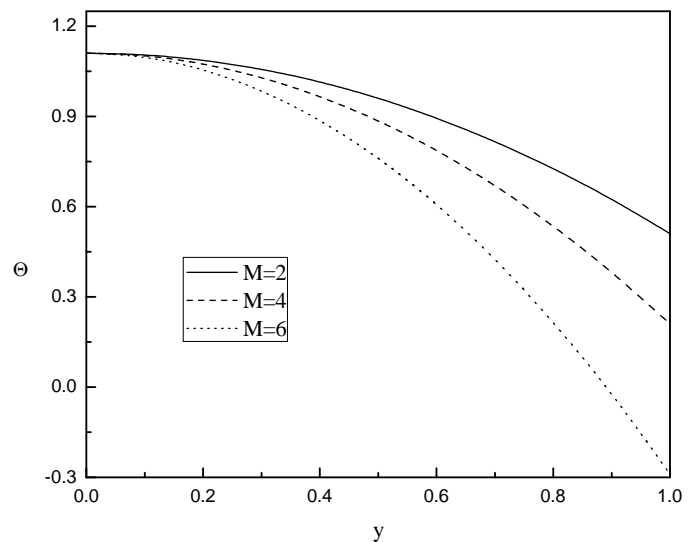

Figure 21: Temperature field for different values of $M$, the other parameters are

$\phi=0.6, G_{r}=3, y_{0}=0.2, D_{a}=0.1, m=1$ and $\alpha=0.01$

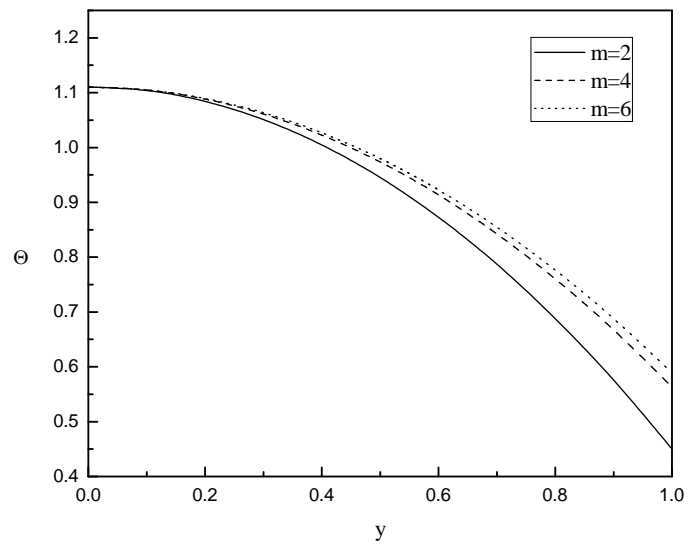

Figure 22: Temperature field for different values of $m$, the other parameters are

$\phi=0.6, G_{r}=3, y_{0}=0.2, D_{a}=0.1, M=4$ and $\alpha=0.1$

\section{REFERENCES}

1. T. W. Latham, M.S. Thesis. MIT. Cambridge. (1966).

2. N.T.M. Eldabe, M.O Shanker and S. A. Maha, Journal of Nanofluids. 7, 595 (2018).

3. Q. Hussain, T. Latif, N. Alvi and S. Asghar, Results in Physics. 9, 121 (2018).

4. R.E.Abo Elkhair, Kh.S Mekheir and A.Z. Zaher, BioNano Science. 8, 596 (2018).

5. S. Nadeem and N. S. Akbar, Comm. Nonlinear Sci. Numer. Simul. 14, 3844 (2009).

6. S. Sreenadh, C. Uma Shankar and A. Raga Pallavi, Int. J. of Appl. Math and Mech. 8, 93 (2012).
7. N. S. Akbar, T. Hayat, Nadeem and S. Obaidat, Int. J. of Heat and Mass Transfer. 55, 1855 (2012).

8. M. M. Bhatti, A. Zeeshan, R. Ellahi and G. C. Shit, Advanced Powder Technology. (2018).

9. Kh. S. Mekheimer and Y. A. Elmaboud, Int. J. of Heat and Mass Transfer. 126, 790 (2018).

10. Kh. S. Mekheimer, S. Z. A. Husseny and Abd Y. Elmaboud, Numer. Methods Partial Diff. Eqs. 26, 747 (2010).

11. S. Nadeem, T. Hayat, N.S. Akbar and M. Y. Malik, Int. J. Heat Mass Transfer. 52, 4722 (2009).

12. F.M. Abbasi, Maimoona Gul and S. A. Shehzad, Physica E: Low-dimensional systems and Nanostructures. 99, 275 (2018).

13. N. S. Gad, Applied Mathematics and Computation. 217, 4313 (2011).

14. Q.Hussain, T.Hayat, S.Asghar and F.Alsaadi, J.Mech.Med.Biol. 1650047 (2015).

15. R, Ellahi, M. M. Bhatti, Int J Numer Methods Heat Fluid Flow. 26, 1802 (2016).

16. Laxmi Devindrappa, N. B. Naduvinamani, Journal of applied science and computations. 5, 908 (2018).

17. Laxmi Devindrappa, N. B. Naduvinamani, International Journal of Scientific research and reviews. 7, 179, (2018).

18. S.K. Asha, G. Sunitha, Journal of Taibah University for science. 13, 155 (2019).

19. V.P. Rathod, D. Laxmi, International journal of Biomathematics. 7, 1450060 (2014).

20. Laxmi Devindrappa, N. B. Naduvinamani, G.C. Shit, International journal of Innovative Technology and Exploring Engineering. 8, 1324 (2019).

21. Laxmi Devindrappa, N. B. Naduvinamani, Journal of Information and Computational Science. 9 (2019). 